\title{
A new transparent beam profiler based on secondary electrons emission for hadrontherapy charged particles beams
}

C. Thiebaux', G. Blain², B. Boyer', E. Delagnes ${ }^{3}$, Y. Geerebaert', O. Gevin ${ }^{3}$, F. Haddad ${ }^{2,4}$, C. Koumeir ${ }^{4}$, F. Magniette ${ }^{1}$, P. Manigot ${ }^{1}$, N. Michel ${ }^{2}$, F. Poirier ${ }^{4}$, N. Servagent ${ }^{2}$, T. Sounalet ${ }^{2}$, M. Verderi ${ }^{1}$

${ }^{1}$ Laboratoire Leprince-Ringuet CNRS-Ecole polytechnique/Palaiseau/France, ${ }^{2}$ Laboratoire SUBATECH, IMT Atlantique CNRS-Université de Nantes/Nantes/France, ${ }^{3}$ IRFU, CEA Université Paris-Saclay/Saclay/France, ${ }^{4}$ GIP ARRONAX/Saint-Herblain/France

Context

Hadrontherapy dose delivery requires continuous and precise measurement of beam properties, intensity, position, and profile with a minimal beam perturbation. PEPITES project aims at developing a new ultra-thin profiler with a good resistance to radiation and operating in the beam line vacuum.

\section{Goal}

- Continuous beam monitoring during patient treatment

- Simple operation and long detector lifetime

\section{Challenges}

- Minimum beam perturbation

- Material budget < 15 $\mu \mathrm{m}$ WET

- Continuous monitoring

- Good radioresistance (up to $10^{8} \mathrm{~Gy} /$ year)

\section{Detector layout}
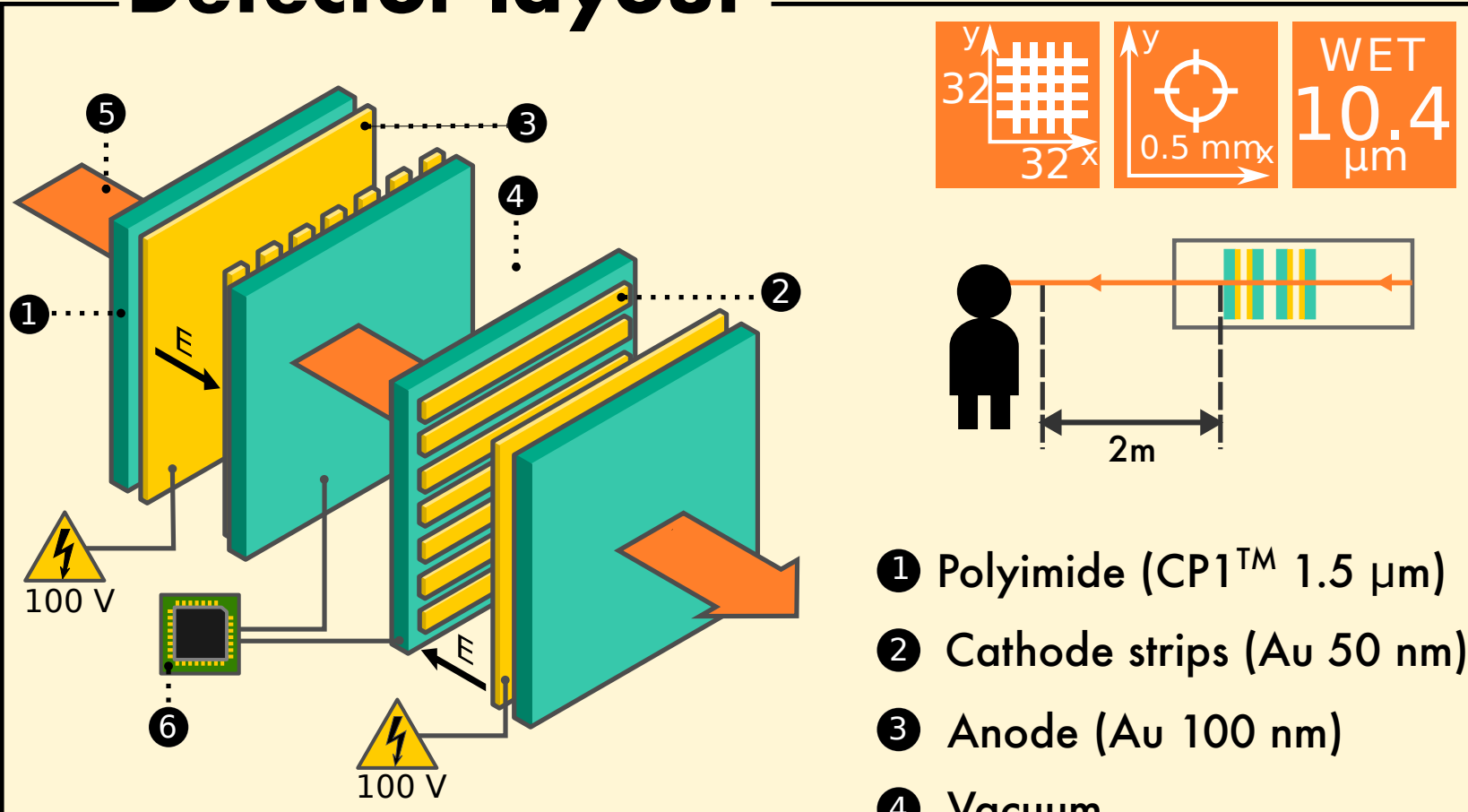

(1) Polyimide $\left(\mathrm{CP} 1^{\mathrm{TM}} 1.5 \mu \mathrm{m}\right)$

(2) Cathode strips (Au $50 \mathrm{~nm}$ )

3 Anode (Au $100 \mathrm{~nm}$ )

(4) Vacuum

5 Incident beam

$C P 1^{T M}$ : www.nexolvematerials.com

6 Readout electronic

\section{Results}

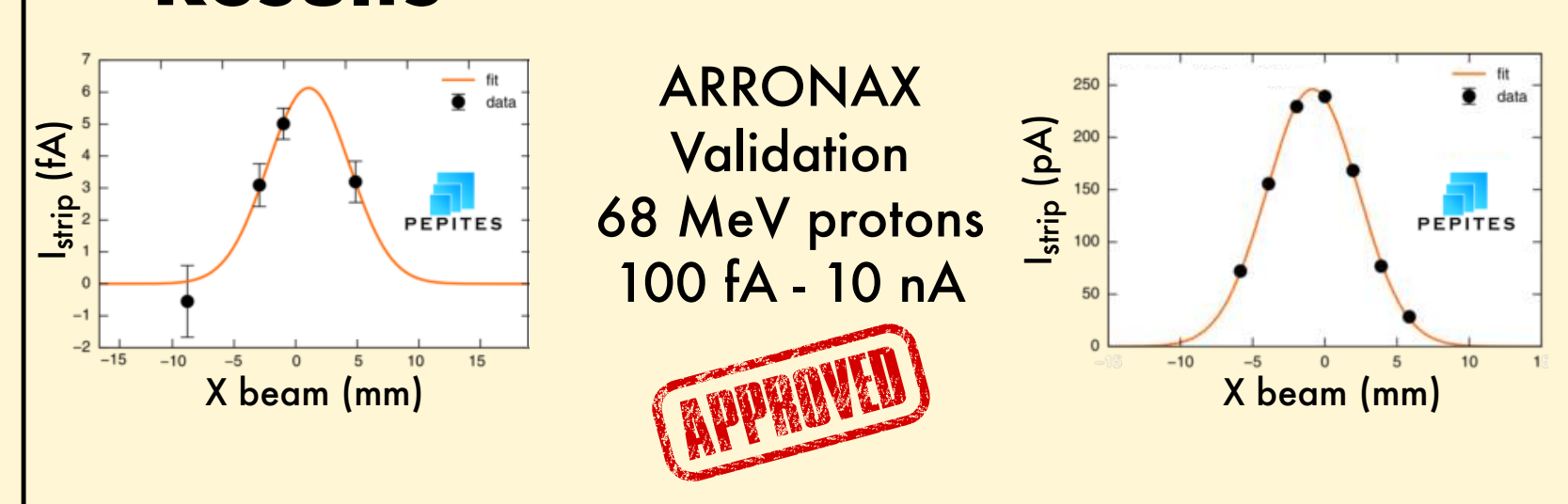

Irradiation with electrons, protons, gamma sources no critical damage up to $10^{9} \mathrm{~Gy}$

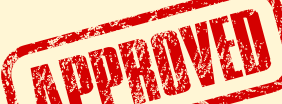

Measurement of SEE

- ARRONAX protons (30-68 MeV) and alphas

- CPO protons (100-230 MeV)
Solution

- Secondary Electron Emission (SEE) for signal - Surface phenomenom

- Sensitive area - $50 \mathrm{~nm}$ gold strips - deposited on $1.5 \mu \mathrm{m}$ polymeric membrane

- Built with thin-film techniques - Modular design

- Adapt patterns to beam specificities

- Operate in beamline vacuum - No mechanical constraints

\section{Prototype}

- Proof of concept - ARRONAXa

- SEE studies

- ARRONAX

- $\mathrm{CPO}^{\mathrm{b}}$

- Hardness studies

- LSI ${ }^{\mathrm{C}}$

- CSNSMd

- ARRONAX

aww.cyclotron-nantes.fr

bOrsay Protontherapy Center

cportail.polytechnique.edu/lsi/fr $d_{w w w . c s n s m . i n 2 p 3 . f r}$

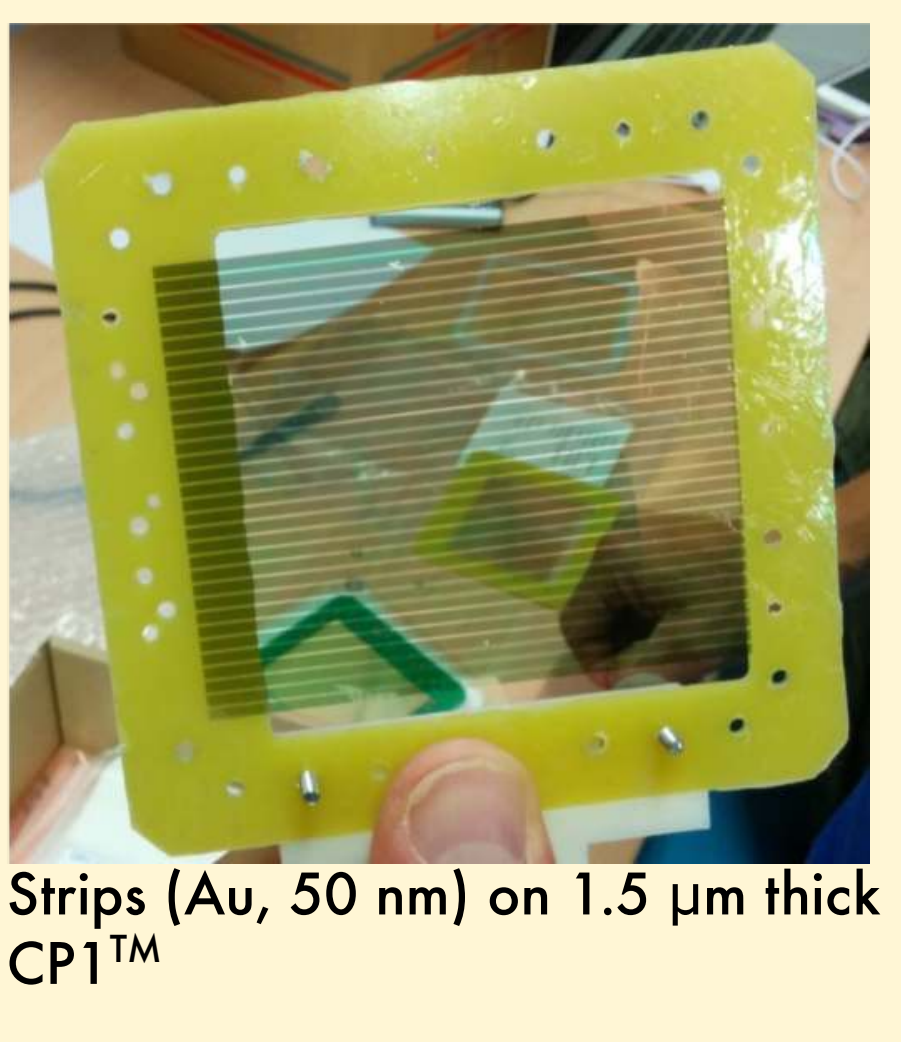

\section{Future}

Inid
2020
end
2020 prototype at ARRONAX cyclotron and operate fully working
2021 Maturate project for PT centers

PEPITES project is supported by the Agence Nationale de la Recherche, ANR-17-CE31-0015, ANR-11-EQPX-0004 and the LABEX P2IO.

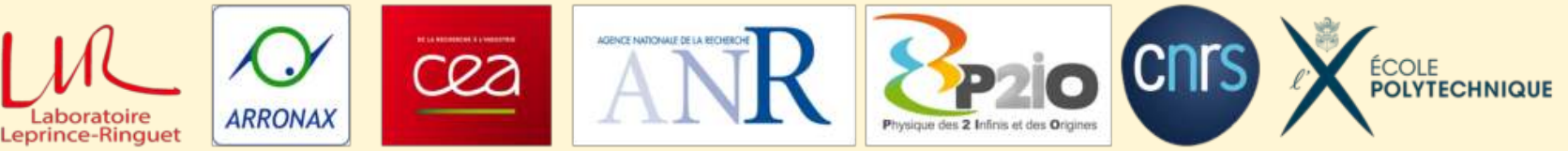

S: Secondary electron

P. Proton

A: Aroton

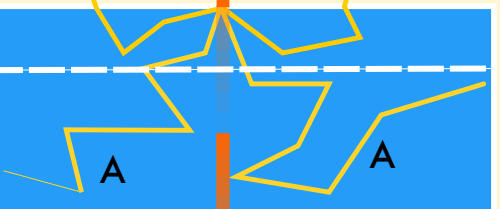

Strips $(A v, 50 \mathrm{~nm})$ on $1.5 \mu \mathrm{m}$ thick 